


\title{
Hacia un destino desconocido: el Memorial de la Shoah en Milán, Italia
}

\author{
Tommaso Cigarini \\ Investigador independiente \\ Italia-Perú \\ tommasocigarini@gmail.com
}

\begin{abstract}
Resumen
El Memorial de la Shoah en Milán es un buen ejemplo de museografía contemporánea. En este documento se analiza la importancia de este tipo de museos para aclarar los periodos más oscuros de nuestra reciente historia y, así, enseñar valores de paz a las generaciones futuras. Si bien la museografía de este Memorial nos cuenta el cruel relato de las deportaciones de judíos y presos políticos durante la Segunda Guerra Mundial, también el edificio de la estación de trenes es en sí una pieza de museo, porque conserva las huellas de las deportaciones en su misma estructura. Es uno de los pocos casos en Europa en donde coinciden contenedor, museo y contenido museográfico.
\end{abstract}

Palabras clave: Shoah, indiferencia, memorial, memoria, museo, museografía.

\begin{abstract}
The Memorial of the Shoah in Milan is a good example of contemporary museography. This document analyzes the importance of this type of museums to clarify the darkest periods of our recent history and, thus, teach values of peace to future generations. Although the museography of this Memorial tells us the cruel story of the deportations of Jews and political prisoners during the Second World War, the building of the train station is itself a museum piece, because it keeps the traces of deportations in its structure. It is one of the few cases in Europe where container, museum and museum content coincide.
\end{abstract}

Keywords: Shoah, indifference, memorial, memory, museum, museography. 


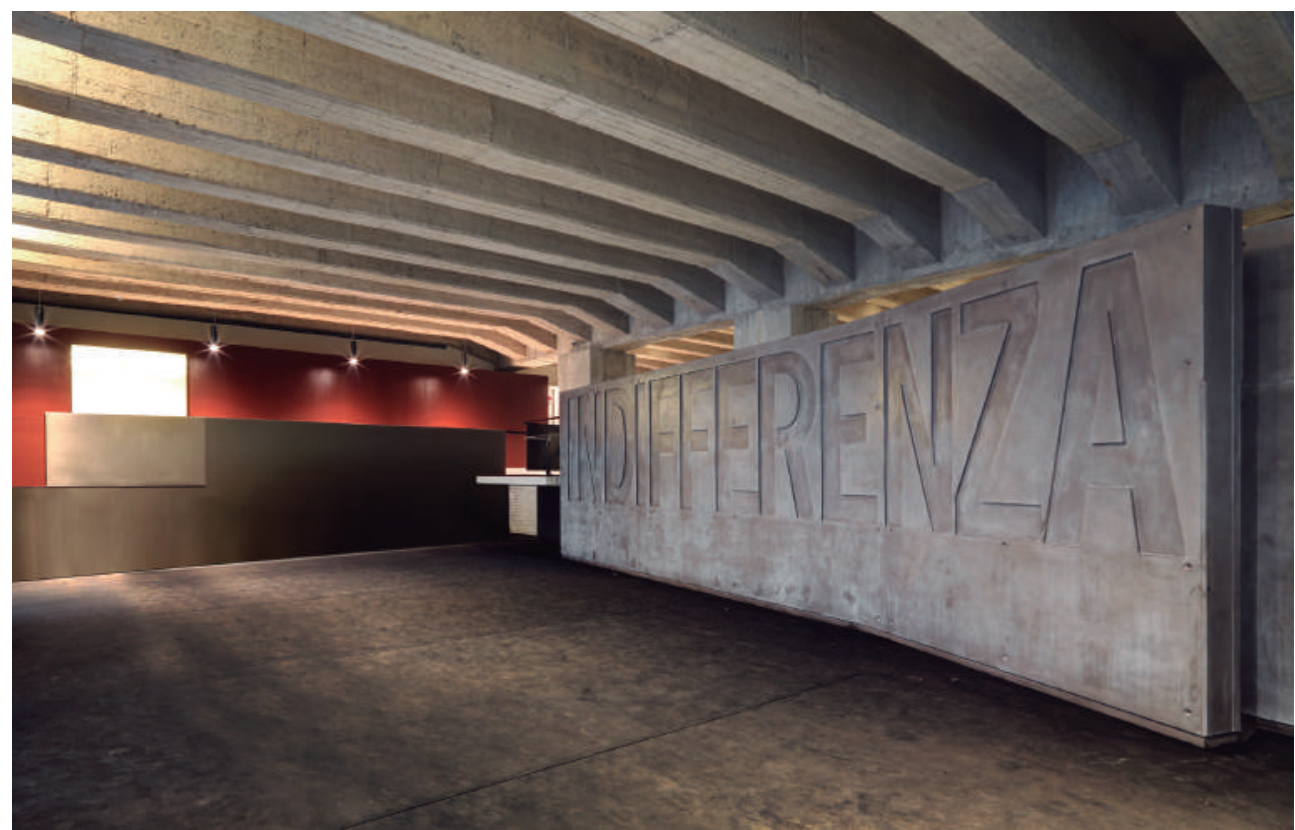

Fig. 1. El hall de ingreso con el Muro de la Indiferencia. Foto: Andrea Martiradonna

En el panorama internacional de la museografía contemporánea, hay que señalar un museo de gran interés recientemente construido ${ }^{1}$ en la ciudad de Milán. Se trata del Memorial de la Shoah, ubicado debajo de la estación de trenes Milano Centrale. El museo ocupa una gran área rectangular de $7060 \mathrm{~m}^{2}$ en el primer piso a lo largo del lado este de la estación de trenes (Fig. 2: planta). ¿Por qué se escogió este lugar para construir un museo dedicado a la Shoah² ${ }^{2}$ La elección del lugar no podía ser más apropiada y emblemática. Desde aquí, durante la Segunda Guerra Mundial, partieron 23 trenes de deportados. Desde 1943 hasta 1945, los nazis, que habían ocupado parte de Italia, en colaboración con las autoridades fascistas italianas, obligaron a subir en trenes de carga a miles de judíos y presos políticos. Se trata del último acto de una cadena de persecuciones contra los judíos y los opositores políticos iniciadas durante la década de 1930 y culminadas con las deportaciones.

A través de una plataforma elevadora, los vagones subían al nivel de los trenes para ser luego ensamblados hasta formar un tren completo y partir rumbo a un "destino desconocido". En realidad, el destino eran los campos de concentración y de exterminio de la Alemania nazi: Auschwitz-Birkenau, Mauthausen, Bergen Belsen, entre otros. Casi todos los deportados murieron en estos campos. De dos trenes que trasladaban 774 judíos y que se fueron directamente a Auschwitz, por ejemplo, sobrevivieron solo 23 personas. ¿Cómo puede la museografía expresar un dolor tan grande en el mismo lugar de las deportaciones? La idea de realizar el memorial nace en 2002, gracias a la iniciativa conjunta de varias asociaciones, entre las cuales se encuentra el Centro de Documentación Judía Contemporánea (CDEC), la Asociación de los Hijos de la Shoah, la Comunidad Judía de Milán y la Unión de las Comunidades Judías Italianas. La Municipalidad de Milán dio el visto bueno para la realización de este memorial 60 años después de las deportaciones ocurridas en este lugar.

1 El memorial abrió sus puertas el 27 de enero del 2012 y la biblioteca lo hará hacia finales del 2019.

2 El término shoah, en hebreo, significa "catástrofe". Se refiere al genocidio étnico, político y religioso de los judíos que tuvo lugar en Europa durante el transcurso de la Segunda Guerra Mundial bajo el régimen de la Alemania nazi. 


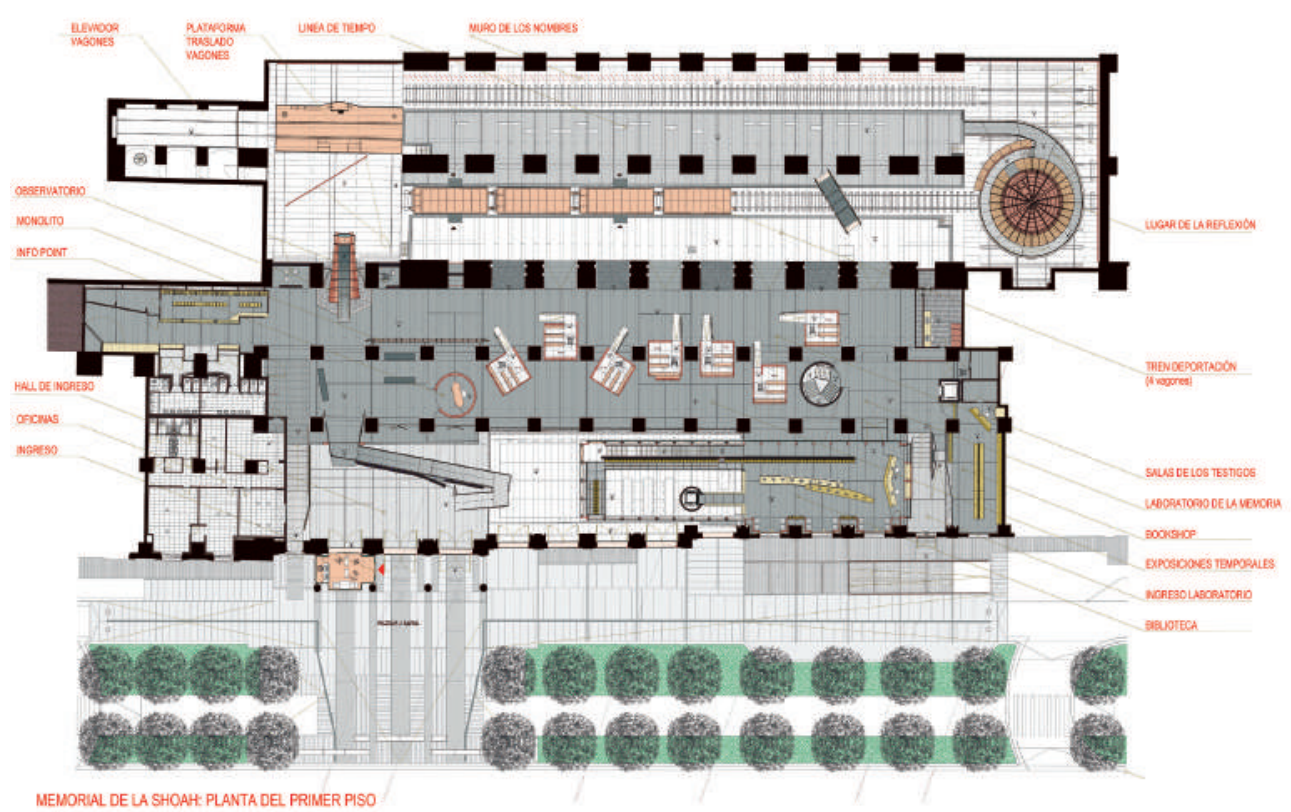

Fig. 2. Planta del primer piso del Memorial de la Shoah. Imagen: Morpurgo de Curtis Architetti Associati

Durante la segunda mitad del siglo XX, gran parte de la sociedad italiana en general no quiso ver ni enfrentar ni asumir el dolor y las responsabilidades de estos sucesos históricos que un memorial tiene que mostrar al público. Es un memorial moderno: no solo un museo, sino también un laboratorio abierto a la ciudad en donde poder conocer y estudiar la cultura judía, así como los sucesos de las deportaciones de la Shoah. Este lugar de la estación de trenes es el único lugar en Europa que ha quedado intacto entre los que han sido usados para las deportaciones de judíos y de presos políticos ${ }^{3}$. El proyecto museográfico del Memorial ${ }^{4}$ es obra del estudio milanés Morpurgo de Curtis Architetti Associati, conformado por Guido Morpurgo y Annalisa de Curtis. Se trata de una pareja de arquitectos que realiza proyectos de todo tipo y escala, siempre con gran atención al contexto y con sensibilidad hacia el espacio, los materiales, el recorrido y el uso de la luz natural.

El primer paso del proyecto del Memorial ha sido el de recuperar el aspecto original del edificio existente que, después de la Segunda Guerra Mundial, había sido modificado, transformándolo en oficinas postales, dividiendo el espacio con tabiques y falsos cielos rasos ocultando casi completamente su aspecto original. Los arquitectos deciden quitar todos los muros y cielos rasos añadidos, dejando desnudas las paredes y estructuras en concreto armado cara a vista. Los muros de concreto, con todas sus imperfecciones y defectos, son así testigos del pasar del tiempo en este lugar. Las estructuras que ahora se pueden ver, columnas y vigas, son enormes, debido a que tienen que soportar las miles de toneladas de los trenes que circulan en el piso de arriba de la estación de trenes (Fig 3: cortes). Los nuevos espacios museográficos, para ser identificables, han sido pensados como objetos en contraste con la materialidad del edificio existente. Son volúmenes de metal, de acero liso,

3 Desde enero del 2017, el museo ha sido vinculado por el Ministerio de Cultura, lo cual implica que no puede ser modificado ni transformado con el tiempo.

4 La oficina Morpurgo-de Curtis reelabora en el 2009 un proyecto preliminar al del 2004 para el Memorial, y se prevé que las obras de la biblioteca, actualmente en curso, terminarán en el 2019. Por lo tanto, entre proyecto y obra, la realización completa del Memorial de la Shoah ha tomado más de 10 años. 


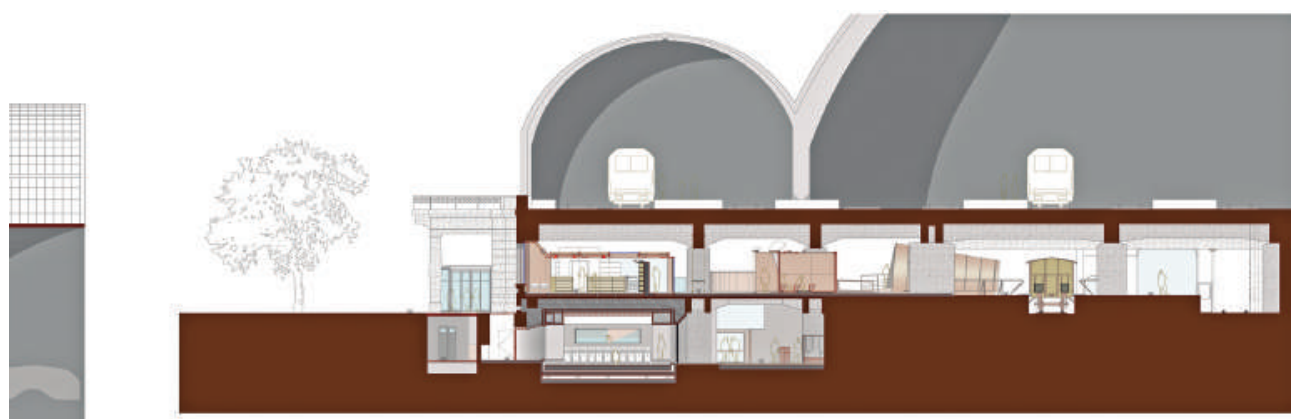

CORTE TRANSVERSAL

EEE

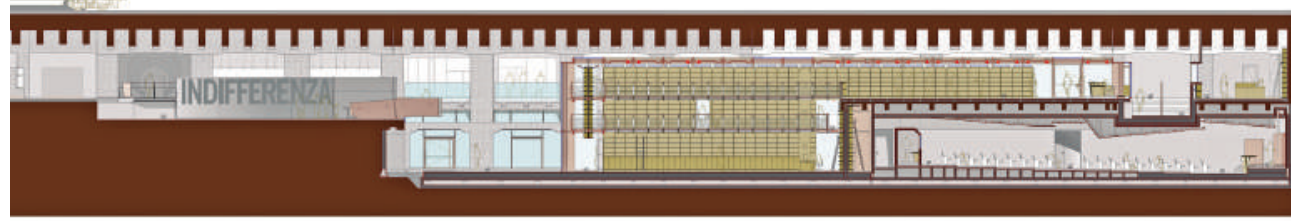

MEMORIAL DE LA SHOAH: CORTES

CORTE LONGTUDINAL

Fig. 3. Cortes transversal y longitudinal del Memorial de la Shoah. Imagen: Morpurgo de Curtis Architetti Associati

muy discretos y esenciales, que se apoyan delicadamente en este gran espacio de concreto. Nunca ofenden el edificio existente, sino que dialogan con él. Es una museografía discreta y fuerte a la vez, que sabe escuchar el peso y el dolor de la historia impregnados en los muros del edificio existente. El museo se articula en dos áreas principales: el "Memorial", zona dedicada al testigo de los eventos; y el "Laboratorio de la Memoria", área dedicada al estudio, la investigación, el encuentro y el diálogo.

El museo ocupa un espacio rectangular de $100 \times 60$ metros, dividido por la estructura del edificio en cinco espacios longitudinales paralelos. La museografía está estructurada por capítulos: el Muro de la Indiferencia, el Observatorio, las Salas de los Testigos, la Vía de la Destinación Desconocida y la Biblioteca "Para no olvidar".

Recorrer el espacio del museo es una experiencia única, que activa todos nuestros sentidos. Por ello, se considera importante relatar la experiencia del visitante al recorrer el Memorial de la Shoah. El ingreso al museo es por el lado este de la estación de trenes, por la plaza E. J. Safra. Una vez que entramos nos topamos con un gran muro de concreto armado que lleva la inscripción "Indiferencia” (Fig. 1). Según los fundadores del museo, los horrores de las deportaciones no hubieran ocurrido sin la indiferencia de la mayoría de la gente. Estamos obligados a bordear ese muro tomando una rampa peatonal a la derecha que nos lleva a un descanso, desde el cual estamos suspendidos encima de un vacío en doble altura, condición parecida a la de las víctimas, que aquí, bajando de los camiones de deportación, ignoraban su triste destino de muerte (Figs. 4 y 5). Superada la rampa y el muro de la indiferencia, vamos descubriendo el horror de las deportaciones, a través de una secuencia de espacios.

El primer espacio que nos llama la atención es el Observatorio. Es un volumen truncocónico horizontal de acero, desde cuyo interior podemos ver, a través de una lupa especial, la plataforma móvil del traslado de los vagones, en ese entonces cargados de deportados (Fig. 6). Una pantalla dispuesta en diagonal nos muestra un documental del 1931, 
que cuenta la historia de la construcción de esta parte de la estación de trenes. El visitante, adentro del cono, está nuevamente suspendido en el vacío, viviendo así la sensación de extrañamiento de los deportados antes de subir a los vagones de los trenes. Del Observatorio pasamos a ver el Monolito: un prisma suspendido de 14 metros de largo en el cual se proyectan videos interactivos con todos los aspectos informativos sobre la Shoah en Italia, con textos y pequeños videos ${ }^{5}$. Luego vemos un espacio longitudinal con las Salas de los Testigos: 6 salas cúbicas de acero de $4 \times 4 \times 3.20$ metros, a las que podemos entrar subiendo pequeñas rampas, sentarnos y ver videoproyecciones con los tristes relatos de los pocos sobrevivientes de las deportaciones. Nos cuentan sus experiencias individuales acerca de la persecución, la deportación y la sobrevivencia al interior de los campos de concentración. Los videos son relatados en diferentes lenguas. Estas salitas son usadas también para proyecciones de videos u obras de arte producidas específicamente durante las exposiciones temporales; asimismo, son usadas para lecturas de textos, pequeños conciertos y espectáculos teatrales. El Piccolo Teatro de Milán ha producido diferentes espectáculos sobre el tema de la memoria en estos espacios. Las salas son presencias silenciosas, pequeños cubos al interior de este gran espacio de concreto. Están ubicadas de manera desfasada y algunas

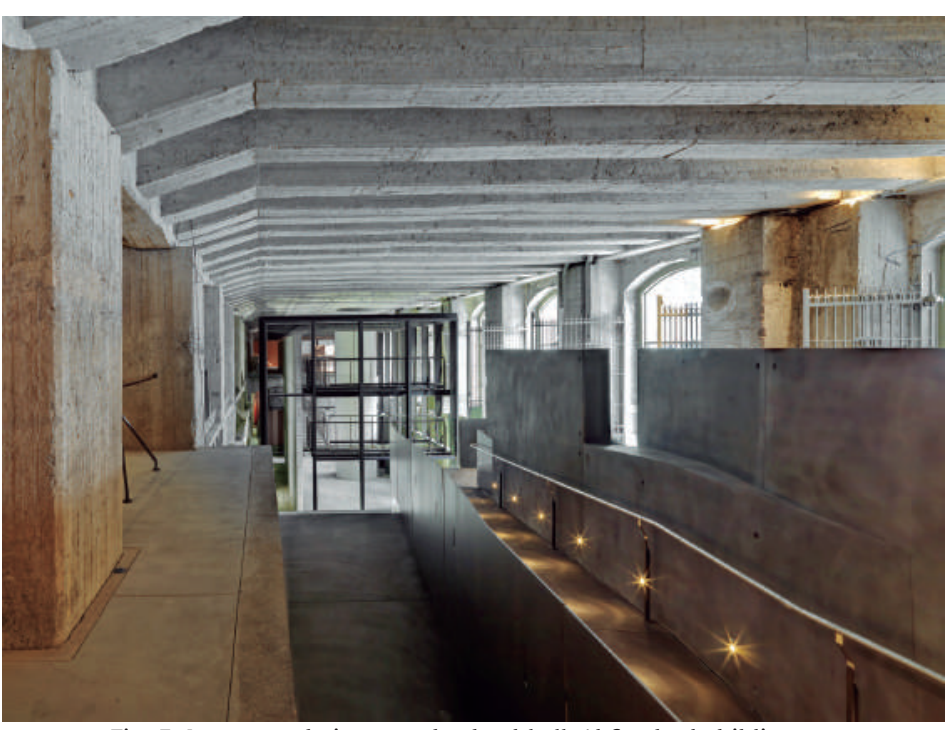

Fig. 5: La rampa de ingreso desde el hall. Al fondo: la biblioteca. Foto: Andrea Martiradonna

son rotadas. Estos desfases dinamizan el espacio, creando un recorrido variado y lleno de sorpresas. Así, estas Salas de los Testigos son variaciones sobre el tema de un volumen

5 Se prevé completar el Monolito dentro de un año. 


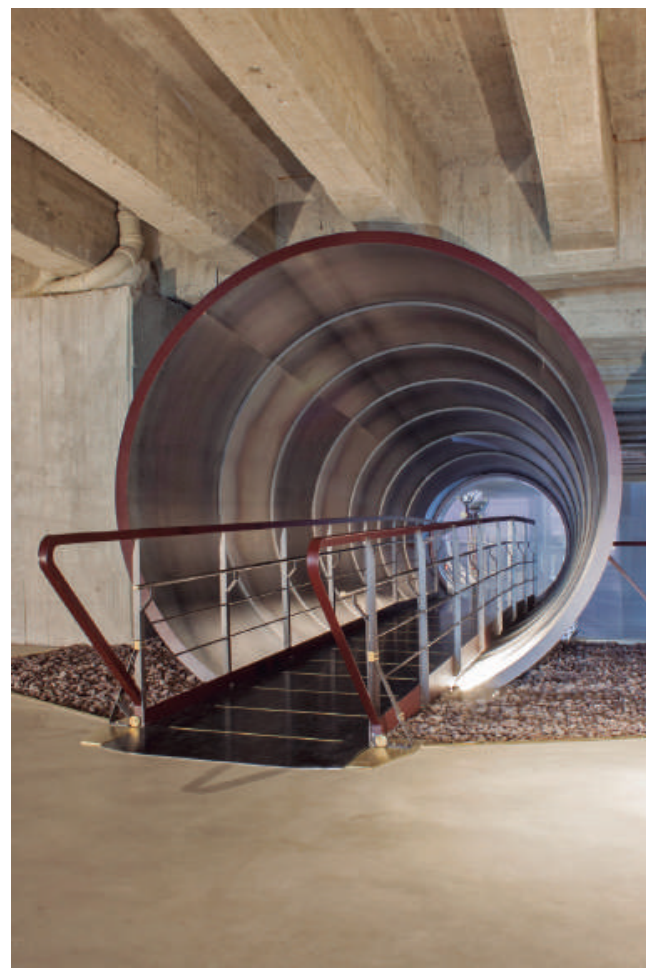

Fig. 6. El Observatorio con vista aérea de los trenes. Foto: Enrico Miglietta virtualmente cúbico. Cada cubo es ligeramente diferente del otro, con cortes diagonales en las paredes y en el techo, los cuales permiten al visitante seguir teniendo un contacto visual con el edificio existente mientras está viendo los videos de los testigos. La fachada posterior al ingreso a cada sala cúbica está equipada con paneles y vitrinas de metal, tensados con cables de acero. Se crea así un espacio de exposiciones temporales a la espalda de los seis cubos dedicadas al tema de la memoria y las deportaciones. El metal frío y los cables tensados de los soportes museográficos transmiten la atmósfera de tensión y soledad que sintieron las víctimas de las deportaciones (Fig.7). El visitante, después de haber tenido un contacto con el dolor de los deportados, puede ahora entrar en el área de las deportaciones, que es un gran espacio rectangular dividido en 2 por grandes columnas, en donde se conservan los rieles y el original tren de madera usado para las deportaciones. Aquí vemos un ejemplar de 4 vagones. El visitante puede entrar en 2 de los 4 vagones y así sentir, aunque de

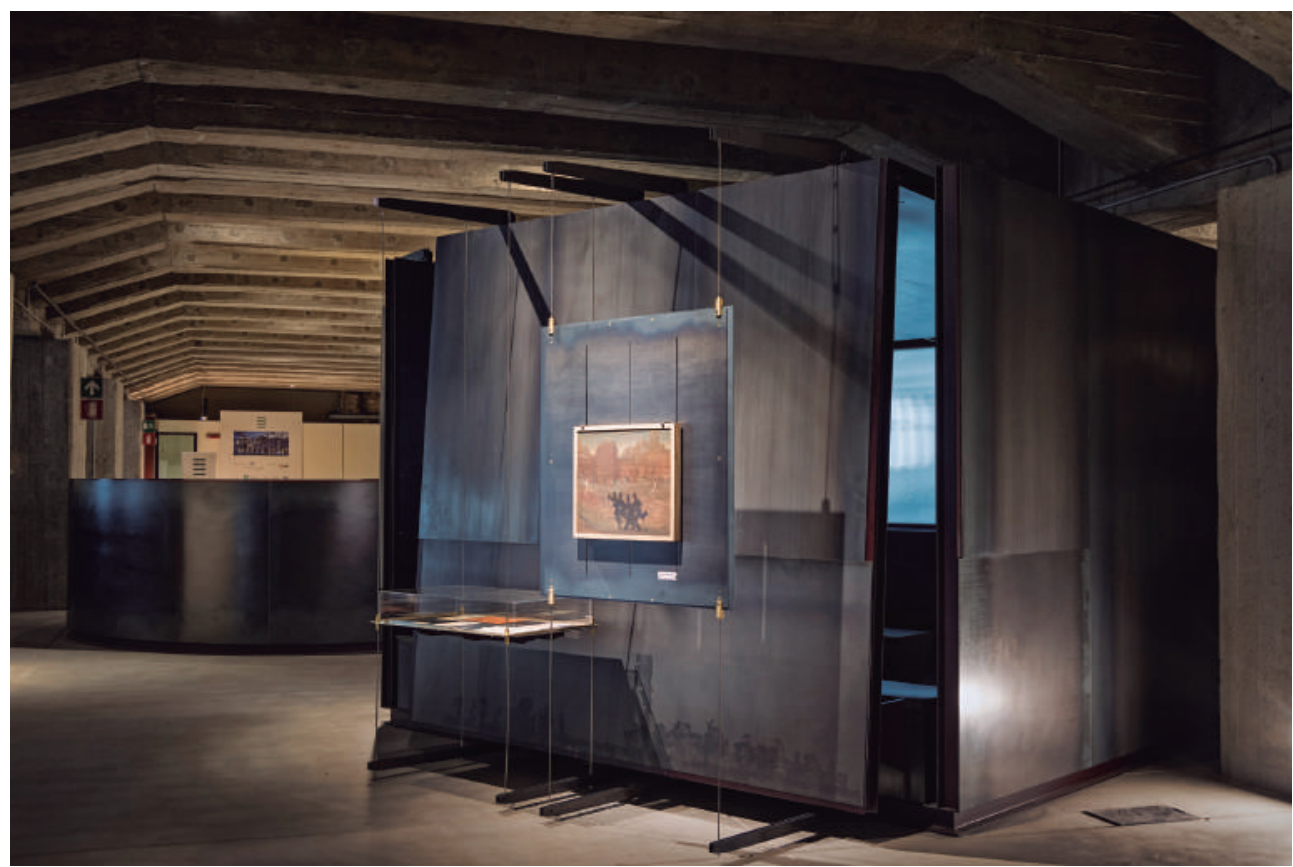

Fig. 7. La Sala de los Testigos, desde la sala de exposiciones temporales para exposiciones temporales. Foto: Paola Mattioli 


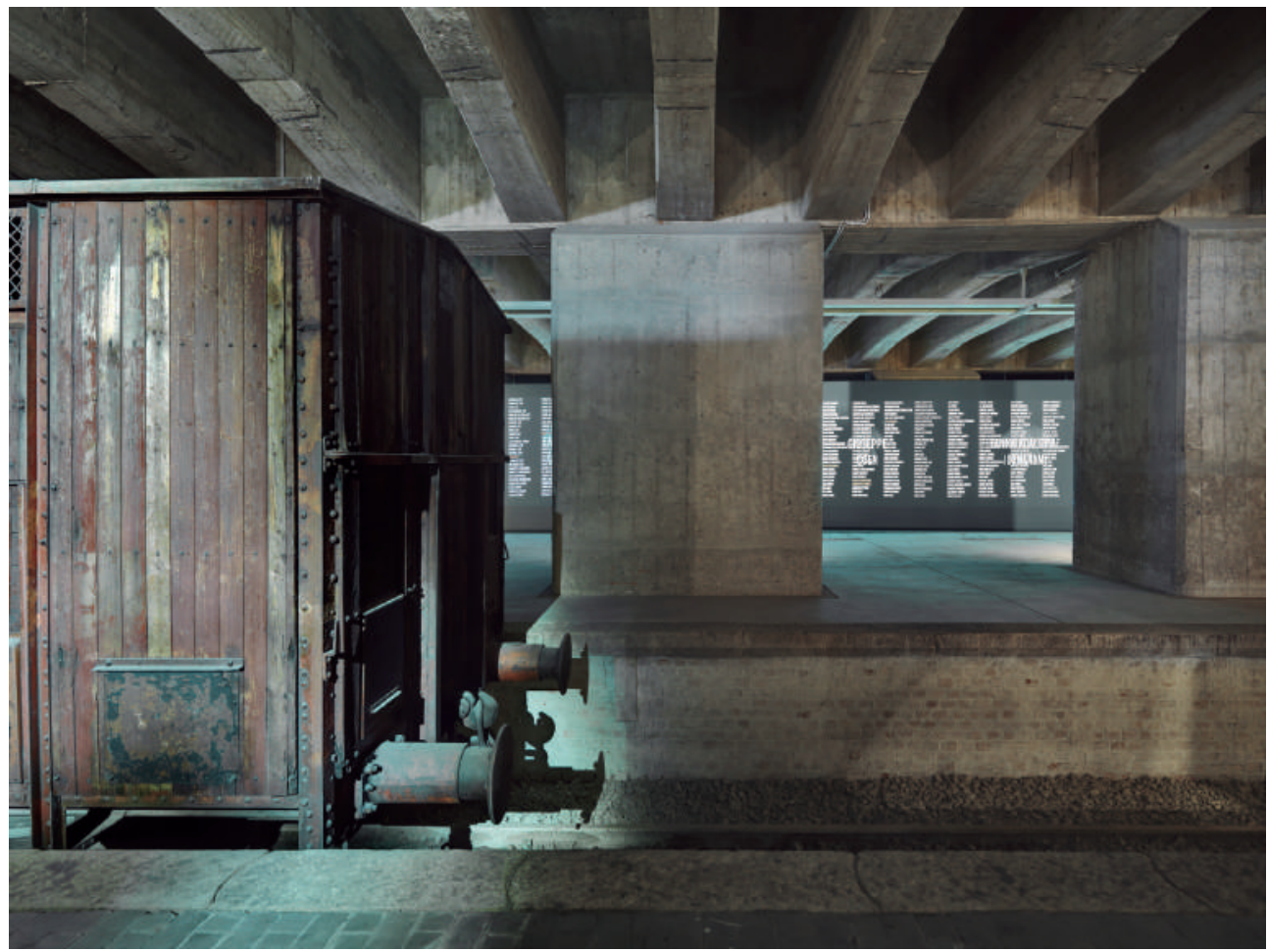

Fig. 8. Detalle del área de la deportación. Al fondo: el Muro de los Nombres. Foto: Andrea Martiradonna

una mínima forma, el miedo, la angustia y la claustrofobia que sintieron las víctimas. Los nazis obligaban a que entre 50 y 60 deportados a la vez suban en cada vagón, sin asientos ni servicios higiénicos, de pie como animales, sin posibilidad de sentarse durante el largo viaje hacia la "destinación desconocida".

Superado este espacio traumático, entramos en un espacio longitudinal con un riel abandonado, sin trenes. Frente a nosotros vemos una gran pantalla blanca llamada Muro de los Nombres, que tiene casi 100 metros de largo (Fig. 8). A lo largo de este muro está proyectada una interminable lista con los nombres y apellidos de todas las personas deportadas desde este lugar, entre diciembre de 1943 hasta enero de 1945. Los nombres de los sobrevivientes aparecen en rojo; fueron en promedio solo el $3 \%$ del total de los deportados. Es un espacio silencioso y oscuro, debido a la lejanía de la luz natural. En el piso de concreto hay planchas de piedra empotradas que llevan inscrita la fecha de la partida de los trenes y el lugar de destinación, así como los diferentes campos de concentración. Es la línea de tiempo de los trenes de la deportación.

El museo se configura así no solo como un contenedor de objetos y piezas, sino como un sistema de espacios que permiten al visitante sentir el drama de la deportación identificándose con las víctimas. Una experiencia tan dolorosa necesita un lugar de descanso. Hacia el extremo norte del museo encontramos el Lugar de la Reflexión, un volumen trunco-cónico vertical de acero que sirve de lugar de meditación, oración o simple descanso visual para el visitante (Fig. 9). El ingreso al volumen no es directo; una rampa en espiral obliga al visitante a girar alrededor del cono antes de entrar en él. Esta experiencia espacial estimula nuestra curiosidad. ¿Qué cosa habrá en su interior? Una vez que entramos nos damos cuenta de que se trata de un espacio completamente vacío, con una banca circular continua en el 


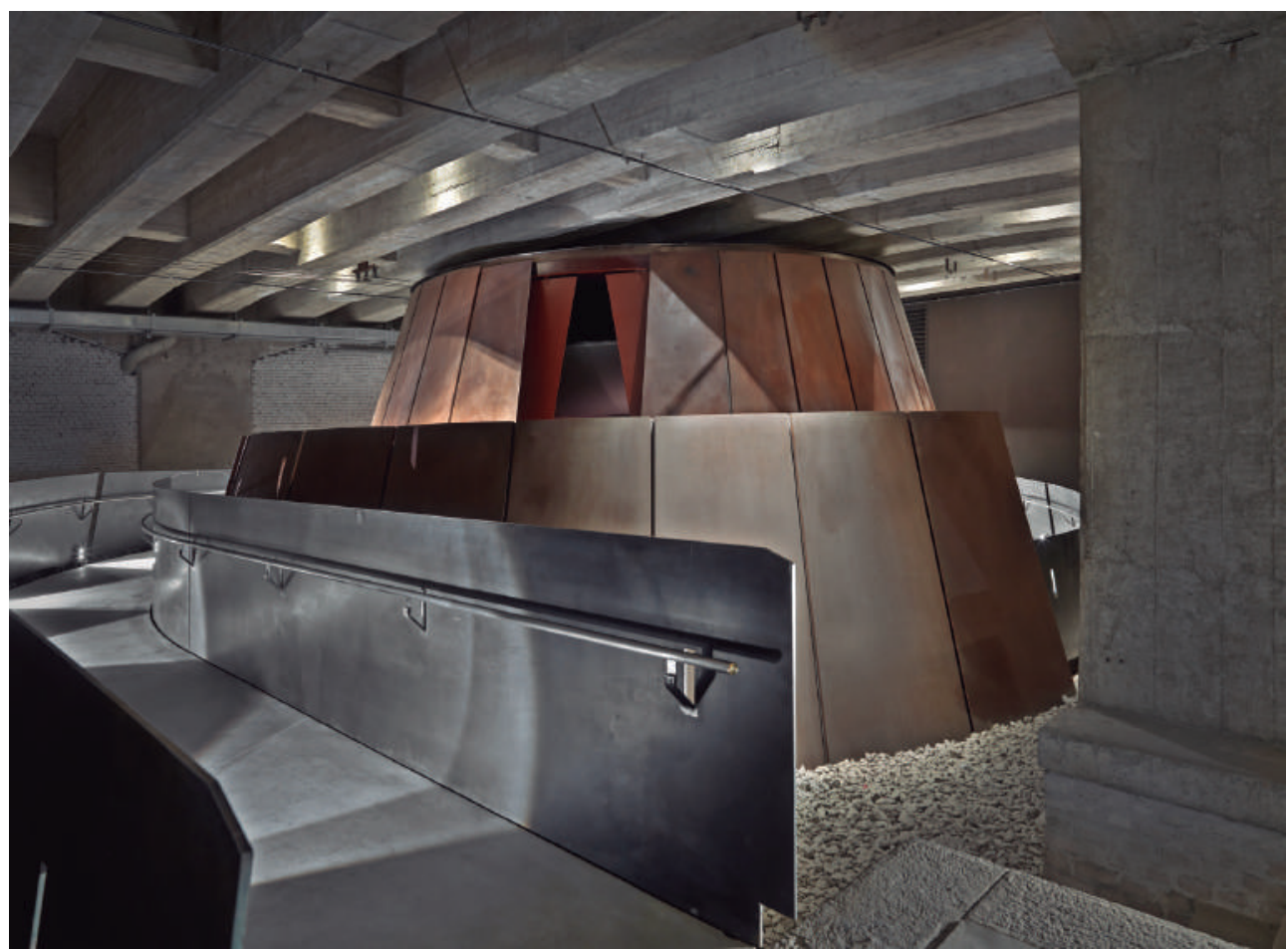

Fig. 9. El Lugar de la Reflexión desde la plataforma del Muro de los Nombres. Foto: Andrea Martiradonna

perímetro. Una vez que nos sentamos nos damos cuenta de que el techo no es plano, sino cóncavo. No podemos distinguir los bordes y los confines de este espacio. En el techo hay una pequeña ventana cilíndrica orientada hacia la ciudad de Jerusalén. Esta ventana ilumina débilmente el piso. Es un vacío que permite calmarnos, recordar lo que acabamos de ver, interiorizándolo con mayor profundidad. Hablando de materialidad, el exterior del Lugar de la Reflexión está hecho con planchas de acero oxidado, mientras que los parapetos de la rampa son de acero en corte natural. El interior está forrado con paneles de acero microforado pintados de rojo oscuro. Este espacio está aislado acústicamente del resto del museo, debido a que está apoyado sobre una especial estructura que absorbe las vibraciones de los trenes del piso de arriba. Acá termina el recorrido del visitante en estos lugares de la memoria y los horrores de la historia.

En el lado noreste del museo hay un segundo ingreso, superado el cual podemos entrar en la zona del Laboratorio de la Memoria. Aquí encontramos, a la derecha, el bookshop del museo; y a la izquierda, el sistema de distribución a la biblioteca y al auditorio. La idea es que el museo sea algo vivo; no solo un lugar de exposición, meditación y de memoria, sino también un lugar de estudio, documentación, investigación, encuentro y debate. La biblioteca se desarrolla sobre dos niveles: el primer piso y el sótano. Ambos niveles están unidos espacialmente por una doble altura creada cortando la losa del primer piso del edificio existente. La gran altura permite el ingreso de luz natural desde grandes mamparas que abren la vista hacia la calle. La biblioteca es un volumen virtual, de acero y vidrio, que no toca las paredes ni el techo del edificio existente, respetándolo. Toda la biblioteca se apoya sobre un sistema especial de amortiguadores sísmicos que sirven para absorber el $85 \%$ de las vibraciones producidas por los trenes en el piso de superior. Un gran muro de concreto, llamado Muro de los Libros, atraviesa longitudinalmente todo el espacio de la biblioteca. Sirve para sujetar y, al mismo tiempo, esconder la escalera de distribución a la sala lectura, a la vez que 
sostiene una enorme estantería en triple altura, a la cual podemos acceder a través de un sistema de ligeros balcones de acero que garantizan la amplitud del espacio (Fig. 10). Tenemos la impresión de estar totalmente envueltos por los libros. En el primer piso hay un nivel de ingreso, acogida y préstamo de libros; además, hay una mesa diagonal con 9 estaciones de Internet que nos conecta con todos los memoriales y museos del mundo dedicados a la Shoah. En el sótano encontramos la sala de lectura, con largos escritorios y estanterías para libros. Los discapacitados pueden acceder a los 2 niveles a través de un ascensor colocado en un cilindro de concreto liso, que se convierte en una enorme columna expresiva que valoriza la verticalidad del espacio. El techo de acero y vidrio de la biblioteca permite la visión de las vigas de concreto del edificio existente, presencia constante en todos los espacios del museo. La biblioteca acogerá 45000 libros del Centro de Documentación Judío Contemporáneo, disponibles para toda la ciudad. La biblioteca está todavía en construcción, pero se prevé su apertura al público hacia finales del 2019. En el sótano, además de la sala lectura de la biblioteca, encontramos un auditorio para 200 personas, quienes pueden acceder a diferentes actividades, como charlas, conferencias, debates, presentaciones de libros y conciertos (Fig. 11). Entre las recientes conferencias que se han realizado recordamos "EHRI Conference European Holocaust Research Infrastructure” (2017 y 2018), y la "Consultation of Planners of New Jewish History and Holocaust Institutions in Europe", organizada por el United States Holocaust Memorial Museum (2018). Al costado del auditorio se encuentra la Sala de Memoriales, en donde hay conexiones con el network en lugares de memoria

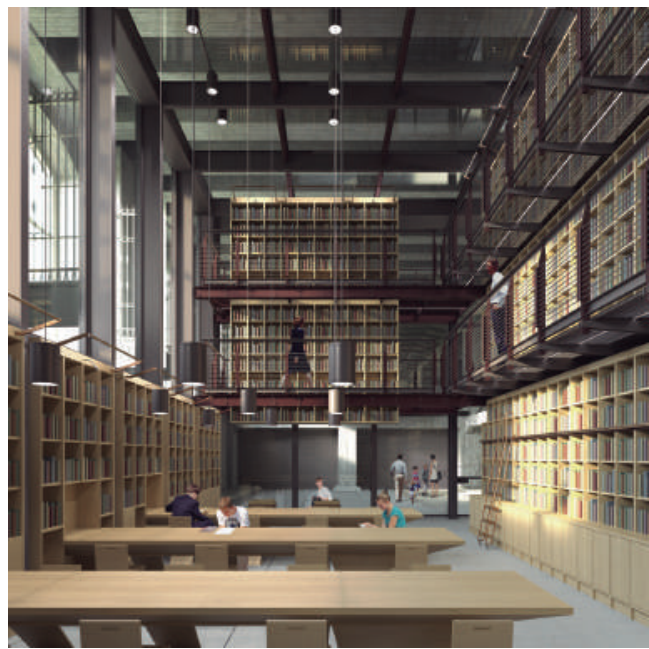

Fig. 10. La sala de lectura de la biblioteca con la triple altura. Rendering de Cavazza \& Pizzuto

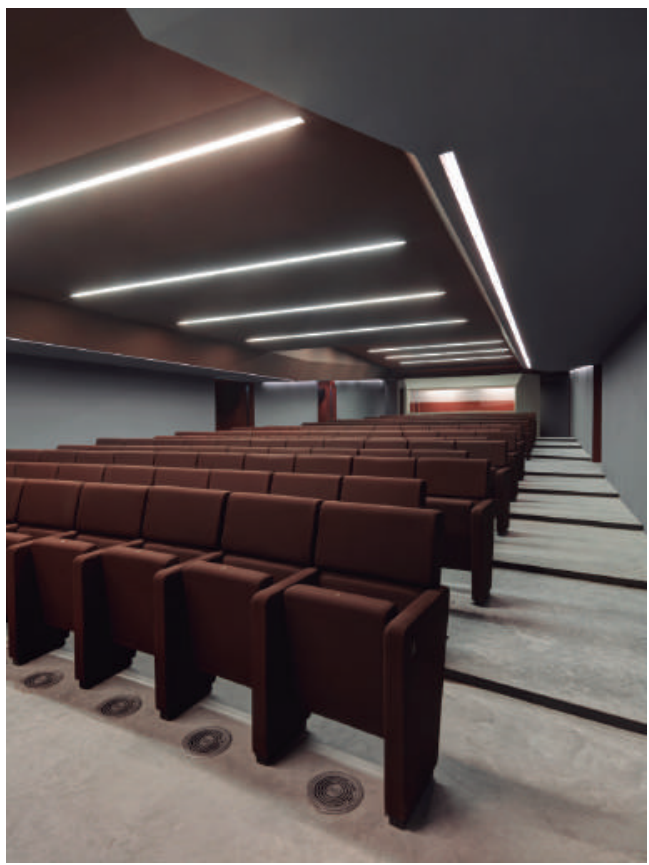

Fig.11. El interior del auditorio en el sótano. Foto: Andrea Martiradonna

sobre la Shoah. Este espacio puede también ser usado como aula didáctica para workshop temáticos.

\section{Conclusión}

El Memorial de la Shoah nos da la oportunidad de interrogarnos sobre la utilidad de los lugares de la memoria. Para quien escribe, la utilidad de estos lugares es enorme, ya que sirven para aclarar y llevar una luz hacia las temporadas más oscuras de nuestra historia reciente, como lo fue la barbarie nazi-fascista. El museo acaba de ganar importantes 
reconocimientos en el mundo de la cultura ${ }^{6}$; sin embargo, en la sociedad democrática italiana de hoy, son varias las tentativas de borrar esta reciente historia, de no querer asumir el dolor y la culpa que conlleva. Esto se manifiesta desde el negacionismo de algunos intelectuales, hasta la arrogancia de algunos grupos parlamentarios que niegan o minimizan las atrocidades de las deportaciones, queriendo así reescribir la historia. Memoriales como este sirven para recordarnos que esto sucedió hace solo 75 años, y sirven para contar a las nuevas generaciones las barbaridades de la guerra que no han vivido ni visto. Asumir el dolor, la memoria y el conocimiento de estos sucesos es el mejor antídoto para que no vuelvan a ocurrir en el futuro.

Mirando hacia la realidad peruana, podemos hacer una comparación entre el Memorial de la Shoah de Milán y el Lugar de la Memoria (LUM) en Lima, analizando afinidades y diferencias. Estos dos museos pertenecen a contextos geográficos y sociales muy diferentes; sin embargo, ambos buscan hacer memoria sobre las barbaridades ocurridas durante una guerra reciente. En el caso del LUM, se trata de la guerra interna desencadenada por Sendero Luminoso desde 1980 hasta el 2000; en el caso del Memorial de la Shoah, se trata de las deportaciones de judíos y presos políticos desde 1943 hasta 1945. El relato museográfico se da con soportes similares, como fotos, objetos o videoentrevistas a los sobrevivientes. Con el tiempo, más familiares de las víctimas o sobrevivientes se animarán a hablar, dejando así su relato de lo vivido en la guerra. Es una cultura inmaterial que va creciendo con el tiempo. Es así que, visitando el LUM, las nuevas generaciones que no vivieron la época del terrorismo, pueden enterarse de lo ocurrido durante la guerra interna, tomando conciencia y evitando así, en el futuro, que estas tragedias se repitan. Las recientes polémicas sobre el LUM, su derecho a existir y su manera de relatar los sucesos del terrorismo, demuestran la actualidad y vigencia de este tema, así como la fuerte fragmentación social que existe en el Perú. Una cultura de paz es posible asumiendo el dolor que la guerra ha causado en las víctimas, iluminando con la memoria los momentos oscuros de nuestro reciente pasado.

\section{Información básica sobre el proyecto}

Nombre del proyecto: Memorial de la Shoah en Milán.

Ubicación: Estación de Trenes “Milano Centrale”, Piazza E. J. Safra, 1.

Área: $7000 \mathrm{~m}^{2}$

Realización: 2009-2019.

Arquitectos: Morpurgo de Curtis Architetti Associati (Guido Morpurgo, Annalisa de Curtis). Fotógrafo: Andrea Martiradonna (principal).

Cliente: Fondazione Memoriale della Shoah di Milano Onlus (Fundación no-profit).

Presupuesto: $€ 12.000 .000$.

\section{Breve nota biográfica de los autores del Memorial de la Shoah}

El estudio Morpurgo de Curtis Architetti Associati, fundado en Milán en 2006, integra las experiencias maduradas por Guido Morpurgo (Milán, 1964; Politécnico de Milán, 1989; PhD, 1999), y Annalisa de Curtis (Milán, 1969; Politécnico de Milán, 1996; PhD, 2004) en la búsqueda de las relaciones entre arquitectura y memoria, entre tradición disciplinar y experimentación. Entre las obras recientes se encuentran dos edificios residenciales en Pioltello, Milán, 2016, y el diseño museográfico de varias exposiciones. Los proyectos realizados por la oficina han sido objeto de exposiciones y publicaciones internacionales. Importantes críticos de arquitectura han escrito acerca del trabajo de Morpurgo de Curtis, entre los cuales se encuentran Marco Biraghi, Graeme Brooker, Beppe Finessi, Vittorio Gregotti, Jacques Gubler, Fulvio Irace y Umberto Riva.

6 El Memorial ha ganado la Mención para la Medalla de Oro por la Arquitectura Italiana, otorgada por la Triennale de Milano (2015). 
Ambos, además, son profesores universitarios en la Escuela de Arquitectura, Urbanismo e Ingeniería de las construcciones del Politécnico de Milán. Han participado en diferentes seminarios internacionales y workshops de taller de proyecto, entre los cuales se encuentran Villard (2014-2018), Polimi Design Summer School (2018), WAVE (IUAV de Venecia 20142015 y 2016) y a la iniciativa "Sketch for Syria" (IUAV, 2016).

\section{Referencias bibliográficas}

Coppa, A. (2018). Memoriale della Shoah, Le sfide dell'architettura 10-Chiese e luoghi di culto e della memoria, Milano: RCS Media Group S.p.A.

Gubler, J. (2018). Dall'invisibile al visibile: la trappola sotto la Stazione Centrale di Milano, in id. "Architettura dell'indelebile. Due Memoriali della Shoah. Milano e Drancy", Milano: Christian Marinotti editor.

Triennale di Milano-Architettura del Moderno, Memoriale della Shoah-Binario 21 (2016). Recuperado de https://www.architetturadelmoderno.it/ luogo/memoriale-della-shoah-binario-21/.

Morpurgo, G. (2016). Architettura e narrazione nel progetto del Memoriale della Shoah: uno scavo archeologico nella Stazione Centrale di Milano, in "ArcHistor. Architettura, Storia, restauro". Recuperado de http://pkp.unirc.it/ojs/index.php/ archistor/article/view/67.

Gregotti, V. (2016). Memoriale della Shoah: al Binario 21 l'architettura dialoga con la memoria, en el diario "Corriere della Sera", Milano.

Irace, F. (2016). L'arte della memoria. Perché questa architettura merita la Medaglia d'Oro, Milano: Editorial "La Repubblica".

Carboni, C.; Colombet, M. \& Rambert, F. (2015). Memorial de la Shoah, Gare centrale, Milan, "Un Bâtiment, combien de vies? La transformation comme acte de création", Paris-Milán: Cité de l'architecture $\&$ de patrimoine-Silvana Editorial.
Andreola, F; Biraghi, M. \& Lo Ricco, G. (2015). Memorial of the Shoah, Central Station, "Milan Architecture Guide 1945-2015", Milano: Hoepli.

de Curtis, A. \& Morpurgo, G. (2015), Il Memoriale della Shoah di Milano: infrastruttura tra documento e progetto, "La freccia del tempo. Ricerche e progetti di architettura delle infrastrutture", Torino: Pearson Editorial.

Suriano, S. (2015). La testimonianza dell'invisibile. Il Memoriale della Shoah di Milano, in "Engramma. La tradizione classica nella memoria occidentale". Recuperado de http://www.engramma.it/ eOS/index.php?id_articolo=2122.

Biraghi, M. \& Micheli, S. (2013). Memoriale della Shoah di Milano, "Storia dell'architettura italiana 1985-2015”, Torino: Einaudi Editorial.

Biraghi, M.; Lo Ricco, G. \& Micheli, S. (2013). Memoriale della Shoah, en "Guida all'architettura di Milano 1954-2014”, Milano: Editorial Hoepli.

Riva, U. (2013). Né un abbandono, né un commento/ Neither an abandonment, nor a comment. Morpurgo de Curtis, Memoriale della Shoah, Milano, revista "Abitare", N. ${ }^{\circ}$ 530, marzo del 2013.

Recibido el 21 de agosto de 2018 Aceptado el 28 de agosto de 2018 